\title{
A trust approach for sharing research reagents
}

\author{
Aled Edwards, ${ }^{1 *}$ Max Morgan, ${ }^{2,3}$ Arij Al Chawaf, ${ }^{1}$ Kerry Andrusiak, ${ }^{2}$ Rachel Charney, ${ }^{2}$ \\ Zarya Cynader, ${ }^{2,4}$ Ahmed EIDessouki, ${ }^{2}$ Yunjeong Lee, ${ }^{2}$ Andrew Moeser, ${ }^{2,4}$ \\ Simon Stern, ${ }^{2}$ William J. Zuercher ${ }^{5}$
}

\begin{abstract}
The core feature of trusts-holding property for the benefit of others-is well suited to constructing a research community that treats reagents as public goods.
\end{abstract}

In 1980, with the passage of the U.S. BayhDole Act, the culture of academic science changed. The act gave universities the responsibility to manage their intellectual property for the betterment of society, with the broad aim of translating more university discoveries into products. In response, academic institutions created technology transfer offices to better manage their potential intellectual property and facilitate technology commercialization (1). Predictably, as part of this more sophisticated approach to technology transfer and partly in response to metrics of success that included numbers of patents, institutions greatly expanded the range of discoveries that they deemed to have potential commercial interest (2). Discoveries were patented at rates not seen previously, and assets such as biomedical research reagents were shared only under legal material transfer agreements (MTAs) that placed restrictions on their commercial and, often, scientific use (3).

MTAs have three main functions. The first is to establish clear "chains of custody," thus assuring the recipient that the material is not encumbered by background agreements or claims. The second is to provide legal protection for the provider. An MTA might include, for example, a clause that states that there is no guarantee that the material is actually safe or fit for particular purposes. The third function is to establish conditions for use of the material. For example, the provider might insert conditions that restrict use or demand claims on future intellectual property.

In aggregate, negotiations over such conditions cause substantial delays in the distribution of research reagents. A university might execute thousands of MTAs each year, with an average delay to execution of a few weeks and with exceptional delays of months and years. As a result, what was born as a solution for the perceived translational gap between academia and industry has evolved into a widely applied and complex system of legal agreements that often encumber even the most basic and commercially irrelevant biomedical research (4). There are now standard template agreements that have streamlined the MTA process, but only a small fraction of reagent exchange is done under these standard MTAs. Examples of such MTAs include the Uniform Biological Material Transfer Agreement (www.autm.net/resourcessurveys/material-transfer-agreements/uniformbiological-material-transfer-agreement) and the U.K. Brunswick MTA (www.arma.ac.uk/ resources-1/research-contracts/material-transferhuman-tissue-agreements).

In another attempt to accelerate science, some projects were established to generate research materials and make them available. Most focus on those materials that have low commercial potential and little chance of generating new intellectual property. For example, the Mammalian Gene Collection project created a repository of sequenced human complementary DNA clones that were made available for a cost but without legal encumbrances (5). Other reagent-generating projects, such as the Structural Genomics Consortium (6) (www. thesgc.org/chemical-probes), provide open access to reagents that have considerable commercial value and that also might be used to generate patentable discoveries. The position of the Structural Genomics Consortium is that these reagents would make a greater impact if kept in the public domain, and the patenting of

\footnotetext{
${ }^{1}$ Structural Genomics Consortium, University of Toronto, Toronto, Ontario M5G 1L7, Canada. ${ }^{2}$ Faculty of Law, University of Toronto, Toronto, Ontario M5S 2C5, Canada. ${ }^{3}$ Grand Challenges Canada, Toronto, Ontario M5G 1L7, Canada. ${ }^{4}$ Gilbert's LLP, Toronto-Dominion Centre, Toronto, Ontario M5K 1K2, Canada. ${ }^{5}$ Structural Genomics Consortium, UNC Eshelman School of Pharmacy, University of North Carolina at Chapel Hill, Chapel Hill, NC 27599, USA.

*Corresponding author. Email: aled.edwards@utoronto.ca
}

discoveries using these reagents would restrict both subsequent scientific discovery and commercial exploitation.

We sought to develop a general mechanism to allow for the widest and least encumbered use of research reagents. The existing standard MTAs restrict the recipient from transferring the material to other scientists, and they also place restrictions on the use of the material commercially. To overcome these limitations, we describe the concept of an "open science trust" as a framework to share research tools. The core feature of trusts-the holding of property for the benefit of othersis better suited than are bilateral contractual arrangements to the construction of a research community dedicated to treating experimental reagents as public goods.

\section{THE TRUST DEVICE}

Under an open science trust, reagents are treated as a public-good resource governed by principles that promote the public interest, in this case, open science. Our open science trust agreement codifies these public-good principles. Under its terms, a recipient of research reagents becomes a "trustee" of the reagents. Trustees are bound by principles that specifically prohibit filing any patent claims that would restrict use of the reagents by others. The result is to create and expand an open science community connected by a common commitment to the foundational aims of the reagent generators.

A trust is a legal relationship whereby one party-called the trustee-is given control over property but must use it for the benefit of others-called the beneficiaries. In this regard, a trust contrasts with direct legal ownership over property, which allows owners to use the property for their own ends and to prevent others from using or benefiting from it. That is how we normally think about tangible goods such as real estate and intangible ones such as patented biomedical inventions.

A trust places a duty on those who possess entrusted assets to manage those assets 
for the benefit of particular third parties or, in the case of charitable trusts, in furtherance of particular objects that benefit the public. Trusts are created by appointing trustees under a legal document that enumerates specific obligations in dealing with trust property. Private truststhose with individual beneficiaries-are often used for tax and estate planning purposes. Charitable trusts, by contrast, are dedicated to serving the public, as opposed to particular individuals, and must have definite charitable objects that guide the trustee's use of trust property. In effect, the "public" constitutes the beneficiary of a charitable trust. Charitable trusts are often administered by a group of trustees whose joint efforts to further the aims of the trust can foster a communal sense of purpose.

\section{THE TRUST AS A TEMPLATE FOR OPEN SCIENCE}

The goal of our project is to provide the community with tools and reagents without restrictions on use. The open science trust concept helps to achieve this end by appointing the recipient of research reagents as a trustee of the reagents, who commits to use them in a manner that serves the trust's beneficiaries. These beneficiaries include (i) the organization that provides the reagents, (ii) others in the scientific community who may benefit from open access to the reagents and the results of the recipient's research, and (iii) members of the general public whose health might be aided by subsequent discoveries.

Whereas it is not, strictly speaking, a charitable trust, the open science trust mechanism seeks to further open science principles for the public good in a similar spirit. Just as a trust document sets out particular conditions aimed at furthering the trust's goals, the open science trust lists a series of specific obligations that the trustee accepts, including (i) the duty to place resulting research and data in the public domain and (ii) the duty not to seek or enforce intellectual property rights. Moreover, because the open science trust is not intended to inhibit downstream innovation, it limits the enumerated obligations to the supplied material itself; the obligations do not extend to derivatives of the material that the trustee might create.

These conditions were crafted to signal to potential recipients that they must embrace open science principles. As a result, the open science trust achieves two objectives. First, it allows a rapid means for researchers to access and share enabling reagents. Second, it aggregates a community of researchers around the reagents, expanding the scope of open science conducted with the reagents. The open science trust differs in form and in spirit from the customary mechanisms to transfer materials between institutions. When an MTA is used to transfer materials, the reagents are treated as commercial goods. Such transactional interactions are often subject to lengthy negotiation and also convey to the recipient a sense of individual ownership of the materials. By contrast, the open science trust fosters a sense of community dedicated to serving the interests of others.

\section{ENFORCING THE TRUST}

Commonly, trusts are enforced through legal mechanisms: private trusts through the threat of lawsuit by the beneficiaries and charitable trusts through the actions of government officials with jurisdiction over charities. In our concept of an open science trust, although its beneficiaries could, in theory, seek redress for violations of the trust conditions, we do not intend for this to occur. We anticipate that the sense of community among recipient trustees, and the norms that are implicit, will become the mechanism to enforce the trust. We believe that the open science trust's provision for sharing results will evolve to become the expected practice by the user community. In this context, precisely because the open science trust model seeks to encourage a sense of communal participation and to inculcate a set of shared open science norms among researchers, a trustee who does not abide by the terms of the trust would potentially face challenges to maintaining good standing within the community. This, we imagine, will provide stronger inducement to adhere to the principles than would the threat of legal action.

The use of community norms to enforce behavior has precedent. Norms, like law, serve to promote cooperation in a community by overcoming socially deleterious but individually rational conduct. Scholars have shown that social norms play an important role in inducing people to follow informal rules in a community. As the property scholar R. Ellickson showed in a study of Shasta County ranchers, members of a community are more likely to obey their own informal laws than the official laws on the books, so long as the community stands behind and enforces those informal laws. In Ellickson's study, ranchers had their own rules concerning responsibility for damage created by escaped cattle, and they used communal sanctions to enforce those rules (7). Normative systems of this nature have the advantage of avoiding the high costs associated with legal enforcement.

We see a strong analogy between Ellickson's ranchers and the biomedical research community. Although research communities are not geographically defined, as in Ellickson's study, they display many of the same features: Members frequently interact both privately and publicly and share much the same sense of mutual dependence, making noncompliant members eligible for various informal sanctions-such as exclusion from conferences and failure to be credited in research articles. These sanctions are likely to be more effective than a remote threat of legal enforcement, given researchers' and their institutions' ongoing need to maintain a profile in the community. Thus, although the trust document might seem to gesture toward a legal enforcement framework as its backdrop, its more salient effect comes from the same set of communal values that the open science trust concept itself seeks to promote.

Laws and norms also influence one another, including in the area of biomedical research. For example, scientific research norms historically promoted science in the public domain, but the Bayh-Dole Act and associated laws have partially subverted these norms in favor of proprietary approaches (8). The open science trust can be seen as an attempt to use law instead to revive, reinforce, and extend the preexisting community norms around open science, specifically in the context of sharing reagents.

\section{A SAMPLE OPEN SCIENCE TRUST AGREEMENT}

For the past 5 years, the Structural Genomics Consortium has used a "click-wrap" MTA (www.thesgc.org/chemical-probes/request) to distribute material with minimal obligations. This MTA is principally designed to protect this consortium against misuse of the reagents. Although this MTA requires the recipient to refrain from filing for intellectual property protection covering the reagents, and has been successfully used to distribute thousands of reagents, it is structured as a bilateral contract and does not create an expanded community of researchers with a shared commitment to open science. The open science trust agreement (OSTA) is the first attempt by the Structural Genomics Consortium to deploy the open science trust mechanism to disseminate research tools (www. thesgc.org/click-trust). In the OSTA, an introductory section first describes the organization, 
creates the trust, and sets out its beneficiaries, which, in this instance, are the Structural Genomics Consortium and its members, researchers in the scientific community, and individuals in the general public who benefit from research with the entrusted material. A series of clauses then outlines the conditions under which the material is transferred. The main differences between a standard MTA and the OSTA are that the OSTA (i) requires the recipient trustee to commit not to file for any intellectual property that covers aspects of the material or its uses; (ii) requires the recipient to place the results from the use of the reagents into the public domain, through open access journals or on openly accessible websites, as rapidly as is possible; and (iii) grants the recipient the right to disseminate the material to additional recipients, provided those recipients agree to the terms of the OSTA.

Most impediments to sharing research reagents, even those of no commercial value, derive not from concerns about safety, research primacy, or the public good but rather from concerns about current and future ownership of intellectual property. In early-stage drug dis- covery research, the morass of agreements and competing intellectual property claims can hinder the progress of science and discovery. We anticipate that the open science trust mechanism will reduce costly redundancies in earlystage research and allow universities and funders to quantify the broader returns on their research investments.

\section{REFERENCES AND NOTES}

1. D. A. Kirby, Creating entrepreneurial universities in the UK: Applying entrepreneurship theory to practice. J. Technol. Transf. 31, 599-603 (2006).

2. Association of University Technology Managers (AUTM), AUTM Licensing Activity Survey Summary: FY2001 Deerfield, IL (AUTM, 2001)

3. V. Rodriguez, Material transfer agreements: Open science vs. proprietary claims. Nat. Biotechnol. 23, 489-491 (2005).

4. T. Bubela, J. Guebert, A. Mishra, Use and misuse of material transfer agreements: Lessons in proportionality from research, repositories, and litigation. PLOS Biol. 13, e1002060 (2015).

5. R. L. Strausberg, E. A. Feingold, R. D. Klausner, F. S. Collins, The mammalian gene collection. Science 286, 455-457 (1999).

6. M. M. Jones, S. Castle-Clarke, D. Brooker, E. Nason, F. Huzair, J. Chataway, The Structural Genomics Consortium: A knowledge platform for drug discovery
(RAND Corporation, 2014); www.rand.org/pubs/ research_reports/RR512.html.

7. R. Ellickson, Order Without Law (Harvard Univ. Press, 1991).

8. A. K. Rai, Regulating scientific research: Intellectual property rights and the norms of science. Northwest Univ. Law Rev. 94, 77-152 (1999).

Acknowledgments: We thank J. Wilbanks for his idea of using a trust mechanism and for fruitful discussions. The open science trust concept was developed as part of a course (K.A., R.C., A.E., and Y.L.) at the Faculty of Law, University of Toronto. Structural Genomics Consortium is a registered charity (\#1097737) that receives funds from AbbVie; Bayer Pharma AG; the Bill and Melinda Gates Foundation; Boehringer Ingelheim; the Eshelman Institute for Innovation; Genome Canada; the Innovative Medicines Initiative (European Union/European Federation of Pharmaceutical Industries and Associations) [Unrestricted Leveraging of Targets for Research Advancement and Drug Discovery (ULTRA-DD) grant no. 115766]; Janssen; Merck \& Co.; Novartis Pharma AG; the Ontario Ministry of Research, Innovation and Science; Pfizer; the São Paulo Research Foundation FAPESP; Takeda; and Wellcome Trust.

10.1126/scitransImed.aai9055

Citation: A. Edwards, M. Morgan, A. Al Chawaf, K. Andrusiak R. Charney, Z. Cynader, A. EIDessouki, Y. Lee, A. Moeser, S. Stern, W. J. Zuercher, A trust approach for sharing research reagents. Sci. Transl. Med. 9, eaai9055 (2017). 


\section{Science Translational Medicine}

\section{A trust approach for sharing research reagents}

Aled Edwards, Max Morgan, Arij Al Chawaf, Kerry Andrusiak, Rachel Charney, Zarya Cynader, Ahmed EIDessouki, Yunjeong Lee, Andrew Moeser, Simon Stern and William J. Zuercher

Sci Trans/ Med 9, eaai9055.

DOI: 10.1126/scitranslmed.aai9055

ARTICLE TOOLS

http://stm.sciencemag.org/content/9/392/eaai9055

RELATED

http://stm.sciencemag.org/content/scitransmed/10/438/eaaq1787.full

REFERENCES

This article cites 5 articles, 1 of which you can access for free http://stm.sciencemag.org/content/9/392/eaai9055\#BIBL

PERMISSIONS

http://www.sciencemag.org/help/reprints-and-permissions

Use of this article is subject to the Terms of Service

Science Translational Medicine (ISSN 1946-6242) is published by the American Association for the Advancement of Science, 1200 New York Avenue NW, Washington, DC 20005. 2017 (C) The Authors, some rights reserved; exclusive licensee American Association for the Advancement of Science. No claim to original U.S. Government Works. The title Science Translational Medicine is a registered trademark of AAAS. 